\title{
Phytoprotection
}

\section{Régie des populations de Cydia pomonella (Lepidoptera : Tortricidea) dans les vergers commerciaux du Québec avec des phéromones de synthèse}

\author{
G. Chouinard, C. Vincent, G. Langlais et M. Roy
}

Volume 77, numéro 1, 1996

URI : https://id.erudit.org/iderudit/706100ar

DOI : https://doi.org/10.7202/706100ar

Aller au sommaire du numéro

Éditeur(s)

Société de protection des plantes du Québec (SPPQ)l

ISSN

0031-9511 (imprimé)

1710-1603 (numérique)

Découvrir la revue

Citer cet article

Chouinard, G., Vincent, C., Langlais, G. \& Roy, M. (1996). Régie des populations de Cydia pomonella (Lepidoptera : Tortricidea) dans les vergers commerciaux du Québec avec des phéromones de synthèse. Phytoprotection, 77(1), 57-64. https://doi.org/10.7202/706100ar
Résumé de l'article

Des diffuseurs à phéromones sexuelles de synthèse ont été installés en 1992 et 1993 dans trois vergers de pommiers (Malus pumila) du Québec, à raison de 1000 diffuseurs ha lors d'un essai de régie des populations de carpocapse de la pomme, Cydia pomonella [Lepidoptera : Tortricidae]. De 1991 à 1992, soit après un an d'essai, les dégâts de carpocapse à la récolte sont passés de $54 \%$ à $32 \%$, de $11 \%$ à $2 \%$, et se sont maintenus à $0 \%$ dans les vergers à forte, moyenne et faible pressions de carpocapse, respectivement. Après deux ans d'essai, les dégâts sont demeurés au même niveau ou ont été diminués davantage selon le type de programme de répression utilisé contre les autres ravageurs. En 1993, une baisse de 75-100 \% des captures a aussi été observée dans les pièges à phéromones par rapport aux captures observées en 1991. 


\title{
Régie des populations de Cydia pomonella [Lepidoptera : Tortricidae] dans les vergers commerciaux du Québec avec des phéromones de synthèse
}

\author{
Gérald Chouinard', Charles Vincent ${ }^{2}$, Guy Langlais ${ }^{3}$ et \\ Michèle Roy ${ }^{4}$
}

Reçu 1995-07-07; accepté 1996-03-31

Des diffuseurs à phéromones sexuelles de synthèse ont été installés en 1992 et 1993 dans trois vergers de pommiers (Malus pumila) du Québec, à raison de 1000 diffuseurs $\mathrm{ha}^{-1}$, lors d'un essai de régie des populations de carpocapse de la pomme, Cydia pomonella [Lepidoptera : Tortricidae]. De 1991 à 1992, soit après un an d'essai, les dégâts de carpocapse à la récolte sont passés de $54 \%$ à $32 \%$, de $11 \%$ à $2 \%$, et se sont maintenus à $0 \%$ dans les vergers à forte, moyenne et faible pressions de carpocapse, respectivement. Après deux ans d'essai, les dégâts sont demeurés au même niveau ou ont été diminués davantage selon le type de programme de répression utilisé contre les autres ravageurs. En 1993, une baisse de 75-100 \% des captures a aussi été observée dans les pièges à phéromones par rapport aux captures observées en 1991.

Chouinard, G., C. Vincent, G. Langlais, and M. Roy. 1996. Population management of Cydia pomonella [Lepidoptera : Tortricidae] in Quebec commercial orchards using synthetic pheromones. PHYTOPROTECTION 77 : 57-64.

Pheromone dispensers were installed in 1992 and 1993, at a rate of 1000 dispensers $\mathrm{ha}^{-1}$, in three Quebec apple (Malus pumila) orchards, to reduce populations of the codling moth, Cydia pomonella [Lepidoptera : Tortricidae]. After one year, codling moth damage at harvest decreased from $54 \%$ to $32 \%$, from $11 \%$ to $2 \%$ and remained at $0 \%$ in the high, medium and low codling moth pressure orchards, respectively. Damage remained at the same level after the second year, or further decreased, depending on the type of spray program used against other pests. Pheromone trap catches in 1993 also decreased from 75 to $100 \%$ when compared to 1991 catches.

1. Centre de recherche et d'expérimentation agricole, Ministère de I'Agriculture, des Pêcheries et de l'Alimentation du Québec, 3300, rue Sicotte, C.P. 480, Saint-Hyacinthe (Québec), Canada J2S $7 B 8$

2. Centre de recherche et de développement en horticulture, Agriculture et Agroalimentaire Canada, 430, boul. Gouin, Saint-Jean-sur-Richelieu (Québec), Canada J3B 3E6. Contribution $n^{\circ} 335 / 96.03 .01 R$

3. Centre d'agriculture biologique de La Pocatière, 401, rue Poiré, La Pocatière (Québec), Canada GOR 1 Z0

4. Centre de recherche et d'expérimentation en régie et protection des cultures, Ministère de I'Agriculture, des Pêcheries et de I'Alimentation du Québec, 2700, rue Einstein, Sainte-Foy (Québec), Canada G1P 3W8 


\section{INTRODUCTION}

Le carpocapse de la pomme, Cydia pomonella L. [Lepidoptera : Tortricidae], est le plus important ravageur des fruits à pépins et à noyau à travers le monde (Riedl 1980). La lutte contre ce ravageur nécessite un programme de surveillance et de traitements à l'aide d'insecticides de synthèse. En l'absence de tels traitements, les dégâts à la récolte en vergers de pommiers (Malus pumila L.) québécois se sont élevés en moyenne à $17 \%$ de 1977 à 1984 (Vincent et Bostanian 1988). D'autre part, l'emploi répété de tels insecticides a causé l'apparition de populations résistantes dans certaines régions des États-Unis (Welter et al. 1991) et de I'Europe (Biliotti 1970; Zlatanova 1990). De façon générale, ces produits sont toxiques pour les ennemis naturels d'autres ravageurs tels que les acariens, les pucerons et les cicadelles (Barnes et al. 1992; Riedl 1980) et constituent un obstacle incontournable pour la mise en place de programmes de lutte intégrée en vergers. Au Québec, certains producteurs de pommes se tournent de plus en plus vers la production de fruits certifiés biologiques ou écologiques et sont intéressés par les méthodes de lutte alternatives aux pesticides de synthèse.

L'utilisation de phéromones sexuelles pour la régie des populations de carpocapse permet d'éviter l'application d'insecticides de synthèse. Les mécanismes impliqués ont été revus par Cardé (1990). Au Québec, cette technique est particulièrement intéressante dans un contexte de production de type écologique. Elle a été expérimentée avec succès contre le carpocapse dans plusieurs régions des États-Unis (Brunner et al. 1992; Pfeiffer et al. 1993), de I'Europe (Charmillot 1990; Trematerra et al. 1993), ainsi qu'au Canada, en Colombie-Britannique (Judd et Gardiner 1991). Des résultats mitigés ont parfois été obtenus avec le carpocapse (Rice et al.1992), et avec d'autres espèces de tordeuses comme Cydia molesta (Bsk.) [Lepidoptera: Tortricidae] (Pree et al. 1994), Argyrotaenia velutinana (WIk.) [Lepidoptera : Tortricidae] (Novak et Roelofs 1985) et Adoxophyes orana F.v.R. [Lepidoptera: Tortricidae] (Charmillot 1981; Charmillot et al. 1981; Van der Kraan et al. 1980). La plupart des échecs ont été reliés au comportement de l'espèce, à l'utilisation d'un produit défectueux, ou encore à l'emploi de la méthode dans des vergers de topographie irrégulière, de petite taille ou d'homogénéité insuffisante.

Le terme " confusion sexuelle " est souvent utilisé pour décrire le phénomène responsable de l'échec de la reproduction dans une atmosphère saturée de phéromone synthétique. Parmi les facteurs qui affectent l'efficacité de la confusion sexuelle figurent les conditions météorologiques, la topographie et le type de vergers rencontrés (Rothschild 1982). Ces facteurs sont représentés au Québec par un climat particulièrement froid et humide, des sols à vergers variant de plats à fortement accidentés, et des plantations de taille et de structure diverses. L'impact de telles conditions sur l'efficacité de la répression du carpocapse à l'aide de phéromones de synthèse n'a encore fait l'objet d'aucune étude.

L'homologation récente au Canada du diffuseur Isomate-C, imprégné de la phéromone synthétique du carpocapse de la pomme, apporte un outil de lutte intéressant pour les producteurs de pommes de tout le pays. Ce produit a été développé et évalué d'abord pour les producteurs de Colombie-Britannique (Judd et Gardiner 1991) en raison de la plus grande importance économique de ce ravageur dans l'Ouest canadien. Au Québec, les producteurs de pommes qui n'utilisent pas les insecticides de synthèse subissent également des pertes importantes causées par le carpocapse. Nous avons donc étudié l'efficacité de la répression du carpocapse à l'aide de phéromones de synthèse sous les conditions climatiques et culturales des vergers québécois, afin de produire des recommandations valables pour les producteurs intéressés.

\section{MATÉRIEL ET MÉTHODES}

Les essais ont eu lieu de 1991 à 1993 dans trois vergers commerciaux du Québec, soit à Saint-Hilaire (lat. $45^{\circ} 34^{\prime} \mathrm{N}$, long. $73^{\circ} 11^{\prime} O$ ), Henryville (lat. $45^{\circ} 7^{\prime} N$, long. $73^{\circ} 13^{\prime} \mathrm{O}$ ) et Rivière-du-Loup (lat. $47^{\circ} 51^{\prime} \mathrm{N}$ long. $69^{\circ} 23^{\prime} O$ ) (Tableau 1). Sauf dans le cas de quelques exceptions notées au tableau 2, les producteurs participant à 
Tableau 1. Caractéristiques des trois vergers expérimentaux utilisés pour étudier la régie des populations du carpocapse de la pomme à l'aide de phéromones de synthèse

\begin{tabular}{|c|c|c|c|}
\hline Caractéristique & Saint-Hilaire & Henryville & Rivière-du-Loup \\
\hline Âge de la plantation & 45 ans & 9 ans & 10 ans \\
\hline Superficie & 2,0 ha & 3,9 ha & 1,2 ha \\
\hline Topographie & légère pente & plat & légère pente \\
\hline Cultivar principal & Mclntosh $(80 \%)$ & Mclntosh (30\%) & Mclntosh (50\%) \\
\hline Type de plantation ${ }^{a}$ & hétérogène & forte densité & forte densité \\
\hline Proximité du verger voisin & $20 \mathrm{~m}$ & $3 \mathrm{~km}$ & $500 \mathrm{~m}$ \\
\hline $\begin{array}{l}\text { Programme de protection } \\
\text { dans le verger voisin }\end{array}$ & aucun & conventionnel & biologique certifié \\
\hline Pression de carpocapse ${ }^{b}$ & forte $(127 ; 54)$ & moyenne $(31 ; 11)$ & faible $(16 ; 0)$ \\
\hline Date d'installation des diffuseurs & $\begin{array}{l}1992-06-08 \\
1993-06-03\end{array}$ & $\begin{array}{l}1992-06-05 \\
1993-06-01\end{array}$ & $\begin{array}{l}1992-06-10 \\
1993-06-10\end{array}$ \\
\hline Cueillette des fruits attaqués ${ }^{c}$ & non & oui & non \\
\hline Date d'évaluation de la récolte & $\begin{array}{l}1992-09-04 \\
1993-08-24\end{array}$ & $\begin{array}{l}1992-09-02 \\
1993-08-20\end{array}$ & $\begin{array}{l}1992-10-23 \\
1993-10-10\end{array}$ \\
\hline
\end{tabular}

a Type standard, forte densité, ou hétérogène (mélange des deux).

b Classification basée sur $(x ; y)$ où $x$ est le nombre moyen de captures de carpocapse par piège, enregistrées durant l'essai dans le verger voisin et $y$ est le pourcentage moyen de fruits attaqués par le carpocapse à la récolte l'année précédant le début de l'essai.

c Cueillette sélective de fruits attaqués par le carpocapse, pratique recommandée en juillet de la première année par Pacific Biocontrol Inc. Iorsque I'historique indique que les populations de départ de carpocapse sont élevées.

Tableau 2. Nombre de traitements insecticides postfloraux appliqués en 1991, 1992 et 1993 , et quantité de fruits affectés par les insectes autres que le carpocapse en période post-florale

\begin{tabular}{|c|c|c|c|c|}
\hline & $\begin{array}{c}\text { Roténone, } B t \\
\text { ( } \mathrm{n}^{\text {bre }} \mathrm{d}^{\prime} \text { applications) }\end{array}$ & $\begin{array}{c}\text { Pyrèthres de } \\
\text { de synthèse } \\
\text { ( } \mathrm{n}^{\text {bre }} \mathrm{d}^{\prime} \text { applications) }\end{array}$ & $\begin{array}{c}\text { Organo- } \\
\text { phosphorés } \\
\text { (n }{ }^{\text {bre }} \mathrm{d}^{\prime} \text { applications) }\end{array}$ & $\begin{array}{c}\text { Dégâts d'insectes } \\
\text { à la récolte } \\
(\%)\end{array}$ \\
\hline \multicolumn{5}{|c|}{ Saint-Hilaire } \\
\hline 1991 & 1 & 0 & 0 & 97 \\
\hline 1992 & 0 & 0 & 0 & 100 \\
\hline 1993 & 0 & 1 & 2 & 5 \\
\hline \multicolumn{5}{|c|}{ Henryville } \\
\hline 1991 & 8 & 0 & 0 & 22 \\
\hline 1992 & 3 & 0 & 0 & 30 \\
\hline 1993 & 4 & 0 & 0 & 37 \\
\hline \multicolumn{5}{|c|}{ Rivière-du-Loup } \\
\hline 1991 & 0 & 0 & 3 & 8 \\
\hline 1992 & 0 & 1 & 0 & 10 \\
\hline 1993 & 0 & 0 & 0 & 10 \\
\hline
\end{tabular}


l'étude ont utilisé un programme de protection minimal de type écologique, et les applications post-florales d'insecticides indispensables à la bonne régie des insectes ravageurs ont été réalisées avec des produits à faible rémanence (roténone, Bacillus thuringiensis). Les interventions préflorales et les interventions de fin de saison (en dehors de la période d'activité des larves et des adultes) ne sont pas rapportées parce qu'elles n'ont aucun impact sur les populations de carpocapse dans les vergers du Québec.

En 1991, le programme de protection ne comprenait pas l'utilisation des diffuseurs de phéromones synthétiques contre le carpocapse. En 1992 et 1993, les diffuseurs Isomate-C (Pacific Biocontrol Corp., Davis, Californie) ont été installés dans le tiers supérieur des pommiers, et à raison de 1000 diffuseurs ha-1 de verger. Chaque diffuseur de type "attache flexible" (longueur : $20 \mathrm{~cm}$ ) contenait $155 \mathrm{mg}$ d'une phéromone synthétique dont la composition était la suivante: $52 \%$ de codlemone [E-8, E-10-dodécadiène-1-ol], $29 \%$ de dodécanol, $6 \%$ de tétradécanol et $13 \%$ de matières inertes. Une densité de diffuseurs deux fois plus élevée a été utilisée dans la rangée périphérique pour contrer l'effet de dilution créé lors de la dispersion de la phéromone (Charmillot 1990). Des sites d'attachement ombragés, situés dans la partie inférieure des bouquets de feuilles, ont été choisis dans chaque pommier de façon à minimiser la dégradation de la phéromone causée par l'exposition à la lumière et aux variations de température (Brown et al. 1992; Rothschild 1982).

Afin de vérifier la capacité des mâles à retrouver les femelles dans un environnement imprégné de phéromones, de un à quatre pièges à phéromone Multi-Pher I (Services Bio-contrôle inc., Sainte-Foy, Qc, Canada) ont été installés entre le 15 mai et le $1^{\text {er }}$ juin à l'intérieur des vergers expérimentaux (Vincent et al. 1990). Les pièges ont été visités à toutes les semaines jusqu'à la fin de la période de vol des adultes (mi-septembre à Henryville et Saint-Hilaire, début août à Rivière-duLoup). Le même dispositif a été utilisé pour le verger situé le plus près de chaque verger expérimental, afin de comparer les captures obtenues dans un envi- ronnement imprégné de phéromones de synthèse à celles obtenues en absence de ces phéromones.

L'efficacité de la méthode a été évaluée par un examen des dégâts apparents sur des fruits $(n=400-900)$ sélectionnés au hasard peu avant la récolte. Pour fins de comparaison, seul le cultivar Mclntosh a été échantillonné dans les vergers expérimentaux et voisins.

\section{RÉSULTATS ET DISCUSS\|ON}

De 1991 à 1992, après un an d'essais, les dégâts de carpocapse à la récolte sont passés de $54 \%$ à $32 \%$ dans le verger à forte pression (Saint-Hilaire), de $11 \%$ à $2 \%$ dans le verger à pression moyenne (Henryville), et sont demeurés indétectables dans le verger à faible pression (Rivière-du-Loup) (Fig. 1). En 1993, la technique a permis de maintenir les dégâts à un niveau comparable à 1992, sauf dans le verger à forte pression, qui n'a présenté aucun dégât alors que le programme de répression contre les autres ravageurs passait du type biologique au type conventionnel (Tableau 2). L'efficacité de la méthode a aussi été révélée par une diminution de $75-100 \%$ des captures d'adultes obtenues dans les pièges à phéromones par rapport aux captures obtenues l'année précédant le début de l'étude (Fig. 2). Durant la même période de 1991 à 1993, les captures cumulées d'adultes de carpocapse en vergers commerciaux du sud-ouest du Québec sont passées de 32 à 26 insectes par piège, soit une baisse de $14 \%$ (Chouinard et Laplante 1993, 1994). Aucun dégât de carpocapse à la récolte n'a été détecté dans ces vergers, mais les dégâts dans un verger témoin sans insecticides sont passés de 23 à $58 \%$ durant la même période (Chouinard et Laplante 1993, 1994).

Dans les vergers expérimentaux ayant conservé un programme de répression de type biologique, soit Henryville et Rivière-du-Loup, I'utilisation de diffuseurs de phéromones en 1992 et 1993 a également permis de réduire le nombre de traitements post-floraux nécessaires pour la répression des insectes tout en conservant une qualité équivalente de fruits pour ce qui est des autres insectes pouvant 


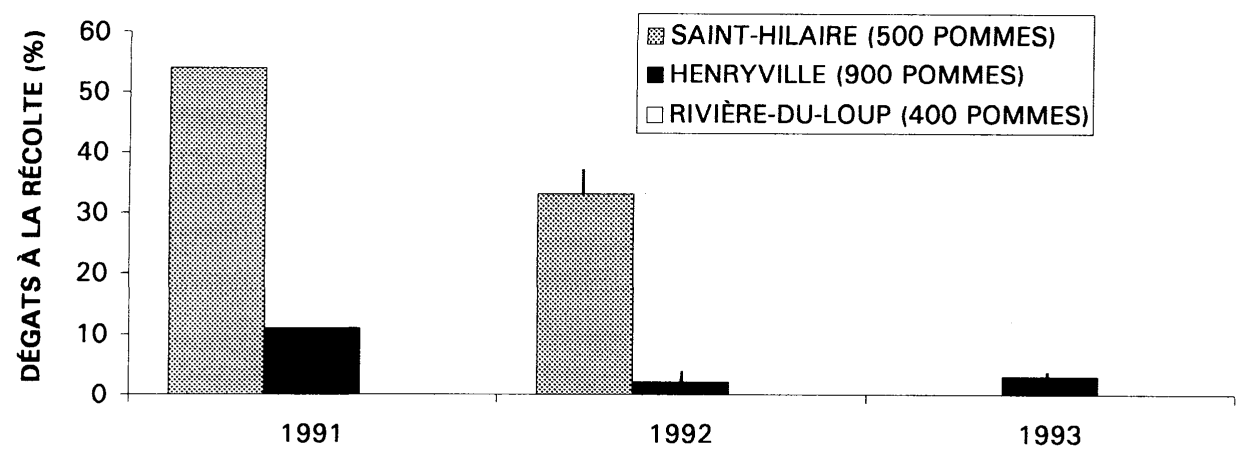

Figure 1. Pourcentage de pommes endommagées par Cydia pomonella à la récolte en 1991 et pendant les essais en 1992 et 1993 dans trois vergers commerciaux du Québec. Les barres verticales représentent l'écart-type mesurant la variation des dégâts entre les 3 sections (nord, centre et sud) de chaque verger.

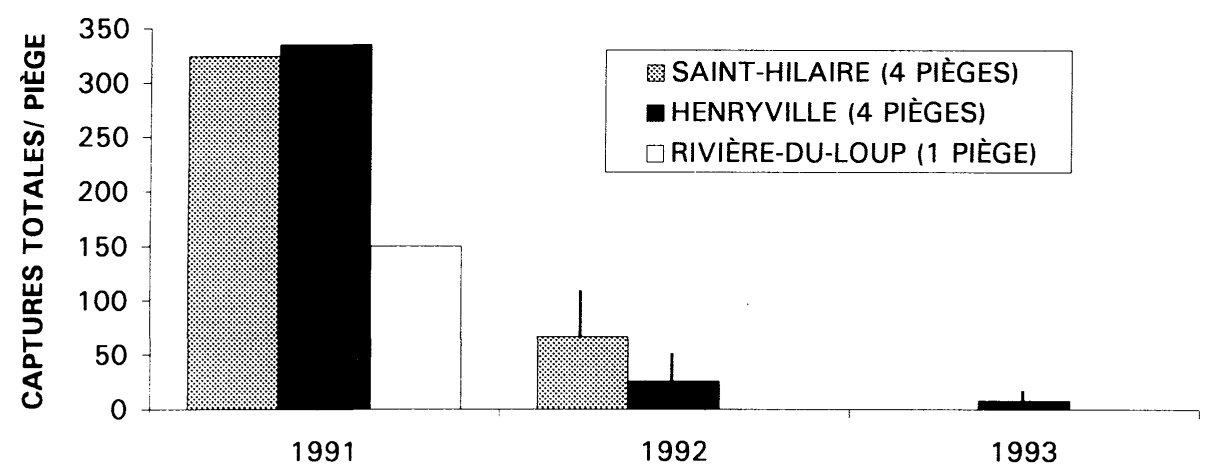

Figure 2. Nombre total de captures de mâles de Cydia pomonella dans les pièges à phéromones en 1991 et pendant les essais en 1992 et 1993 dans trois vergers commerciaux du Québec. Les barres verticales représentent l'écart-type mesurant la variation des captures entre chaque piège. 
causer des dégâts en période post-florale (Tableau 2). De 1991 à 1993, on note une réduction de $50 \%$ dans le nombre de traitements appliqués à Henryville, une réduction de $100 \%$ à Rivière-du-Loup, et une augmentation de 2 à $15 \%$ dans le pourcentage de fruits affectés par ces insectes à la récolte.

L'utilisation seule de phéromones de synthèse pour la répression du carpocapse n'a pas été efficace dans tous les cas. En effet, les dégâts de carpocapse à la récolte s'élevaient à $32 \%$ dans le verger de Saint-Hilaire après la première saison d'essai (Fig. 2). Ceci est supérieur au maximum acceptable d'un point de vue économique qui est de $5 \%$ de fruits endommagés (Vincent et Bostanian 1988). Le manque d'efficacité dans ce cas s'explique de deux façons (Tableau 1). Premièrement, la proximité d'un verger comportant de fortes populations de carpocapse permet le déplacement de femelles accouplées à l'extérieur du verger expérimental. La présence de vergers négligés à moins de $100 \mathrm{~m}$ d'un verger commercial constitue une source reconnue d'infestation par le carpocapse (Myburgh et al. 1975). Deuxièmement, I'hétérogénéité de la plantation favorise la formation d'espaces morts dans le nuage de phéromone, espaces dans lesquels l'accouplement est possible.

La technique s'est avérée efficace dans les autres cas (Figs. 1 et 2), malgré les contraintes suivantes. Le Québec est à la limite géographique nordique de la zone de production, et la température affecte grandement le taux de diffusion de la phéromone, un facteur directement relié à l'efficacité de la technique (Trematerra et al. 1993). La petite taille de plusieurs vergers québécois est aussi un facteur de risque, étant donné que la méthode diminue d'efficacité lorsque les bordures représentent un plus fort pourcentage de la superficie du verger (Charmillot 1990). Finalement, la présence de dénivellations dans plusieurs vergers entourant les collines peut causer un problème d'homogénéité du nuage de phéromone (Rothschild 1982). Nos résultats suggèrent cependant que la méthode mise à
I'essai pourrait s'avérer efficace dans les conditions climatiques du Québec, et ce, pour des vergers de 2-3 ha et plus, pour$\mathrm{vu}$ qu'ils respectent les autres critères mentionnés ci-haut.

$D^{\prime}$ après nos résultats, la répression des populations du carpocapse de la pomme a été réalisée de façon satisfaisante grâce à l'utilisation des phéromones de synthèse, tout en maintenant des rendements et une protection des fruits acceptables d'un point de vue économique. Dans le verger écologique de Henryville par exemple, les coûts d'utilisation en 1993 incluant I'achat et la pose des diffuseurs étaient de 380 \$ CAN ha-1. Le nombre réduit de traitements insecticides postfloraux en 1993 par rapport à 1991 (Tableau 2) a permis d'économiser $365 \$ \mathrm{CAN} \mathrm{ha}^{-1}$ en roténone, tout en conservant le même niveau de répression des ravageurs et en permettant de conserver l'appellation "biologique" de la récolte (O.C.I.A. 1995). D'un point de vue économique, I'utilisation de cette méthode de lutte pour la répression du carpocapse dans ce verger apparaît donc comparable à I'utilisation de roténone. D'un point de vue environnemental, elle est cependant supérieure en raison de sa spécificité.

Cette méthode de lutte intéressera d'abord les producteurs de fruits dits biologiques, qu'ils soient certifiés ou non. Pour ces producteurs, I'utilisation de phéromones de synthèse pour la régie des populations de carpocapse constitue un pas de plus vers l'élaboration d'un programme de répression visant un minimum d'effets néfastes sur l'utilisateur, la culture, le consommateur et l'environnement. Dans la plupart des vergers commerciaux cependant, le carpocapse est contrôlé efficacement par des applications d'insecticides de synthèse et ce, à un coût largement inférieur aux coûts des insecticides naturels comme la roténone, et inférieur également au coût de I'utilisation des phéromones de synthèse. La régie des populations du carpocapse par cette méthode constitue néanmoins, dans tous les cas, une alternative efficace de lutte contre ce ravageur dans les vergers québécois. 


\section{REMERCIEMENTS}

Nous remercions les trois pomiculteurs participants pour les ressources (verger, temps, argent) consenties, ainsi que $\mathrm{Gi}$ nette Laplante et Benoit Rancourt pour la direction technique. Merci également à Julie Boulé pour les informations concernant I'historique du verger à Henryville, et à Jean Larose (ministère de l'Agriculture, des Pêcheries et de l'Alimentation du Québec) pour avoir facilité les démarches administratives. Ce projet a été subventionné par le Programme d'aide à I'innovation technologique en agriculture du ministère de l'Agriculture, des Pêcheries et de l'Alimentation du Québec.

\section{RÉFÉRENCES}

Barnes, M.M., J.G. Millar, P.A. Kirsch et D.C. Hawks. 1992. Codling moth (Lepidoptera: Tortricidae) control by dissemination of synthetic female sex pheromone. J. Econ. Entomol. 85 : 1274-1277.

Biliotti, E. 1970. La lutte intégrée et I'avenir des interventions phytosanitaires. Bull. Tech. Ing. Min. Agric. 249 : 239-246.

Brunner, J.F., L.J. Gut et A. Knight. 1992. Transition of apple and pear orchards to a pheromone-based pest management system. Proc. Wash. State Hortic. Assoc. 88 : 169-175.

Brown, D.F., A.L. Knight, J.F. Howell, C.R. Sell, J.L. Krysan et M. Weiss. 1992. Emission characteristics of a polyethylene dispenser for mating disruption of codling moth (Lepidoptera: Tortricidae). J. Econ. Entomol. 85 : 910-917.

Cardé, R.T. 1990. Principles of mating disruption. Pages 47-71 in: R.L. Ridgeway, R.M. Silverstein et M.N. Inscoe (éds.), Behaviormodifying chemicals for insect management. Marcel Dekker, New York.

Charmillot, P.-J. 1981. Technique de confusion contre la tordeuse de la pelure Adoxophyes orana F.v.R. (Lep., Tortricidae): II. Deux ans d'essais de lutte en vergers. Mitt. Schweiz. Entomol. Ges. 54 : 191-204.

Charmillot, P.-J. 1990. Mating disruption technique to control codling moth in western Switzerland. Pages 165-182 in : R.L. Ridgeway, R.M. Silverstein et M.N. Inscoe (éds.), Behavior-modifying chemicals for insect management. Marcel Dekker, New York.
Charmillot, P.-J., A. Scribante, V. Pont, D. Deriaz et C. Fournier. 1981. Technique de confusion contre la tordeuse de la pelure Adoxophyes orana F.v.R. (Lep., Tortricidae) : I. Influence de la diffusion d'attractif sexuel sur le comportement. Mitt. Schweiz. Entomol. Ges. 54 : 173-190.

Chouinard, G. et G.H. Laplante. 1993. Rapport d'activités, protection des vergers 1992 (réseau pommier). Service de phytotechnie de Saint-Hyacinthe, ministère de I'Agriculture, des Pêcheries et de I'Alimentation du Québec, Saint-Hyacinthe. 136 pp.

Chouinard, G. et G.H. Laplante. 1994. Rapport d'activités, protection des vergers 1993 (réseau pommier). Service de phytotechnie de Saint-Hyacinthe, ministère de l'Agriculture, des Pêcheries et de l'Alimentation du Québec, Saint-Hyacinthe. 151 pp.

Judd, G.J.R. et M.G.T. Gardiner. 1991. Largescale commercial trials of pheromonemediated mating disruption for control of codling moth in British Columbia apple and pear orchards. Agriculture Canada Summerland Research Station, Summerland, B.C., 59 pp.

Myburgh, A.C., H.F. Madsen, D.J. Rust et I.P. Bosman. 1975. Codling moth (Lepidoptera: Olethreutidae): Sex attractant traps as an adjunct to control programs. Pioc. Int. Congr. Entomol. Soc. South Afr. 1 : 99108.

Novak, M.A. et W.L. Roelofs. 1985. Behaviour of male redbanded leafroller moths, Argyrotaenia velutinana (Lepidoptera : Tortricidae), in small disruption plots. Environ. Entomol. 14 : 12-16.

O.C.I.A. 1995. Association pour l'amélioration des cultures biologiques: cahier des charges 1995. Organic Crop Improvement Association, Bellefontaine, Ohio. 63 pp.

Pfeiffer, D.G., W. Kaakeh, J.C. Killian, M.W. Lachance et $P$. Kirsch. 1993. Mating disruption for control of damage by codling moth in Virginia apple orchards. Entomol. Exp. Appl. 67 : 57-64.

Pree, D.J., R.M. Trimble, K.J. Whitty et P.M. Vickers. 1994. Control of oriental fruit moth by mating disruption using sex pheromone in the Niagara Peninsula, Ontario. Can. Entomol. 126: 1287-1299.

Rice, R.E., D.L. Flaherty et W.J. Bentley. 1992. Mating disruption for control of orchard pests in California. Acta Phytopathol. Entomol. Hung. 27 : 525-534.

Riedl, H. 1980. Monitoring and forecasting methods for codling moth management in the United States and Canada. EPPO Bull. $10: 241-252$. 
Rothschild, G.H.L. 1982. Suppression of mating in codling moths with synthetic sex pheromone and other compounds. Pages 118-134 in : A.F. Kydonieus, M. Beroza et G. Zweig (éds.), Insect suppression with controlled release pheromone systems, vol. 2. CRC Press, Boca Raton, Florida.

Trematerra, P., C. loriatti, G. Schreiber et A. Notali. 1993. Mating disruption with semipermeable membrane dispensers for the control of Cydia pomonella L. Pages 277281 in : L.J. McVeigh, D.R. Hall et P.S. Beevor (éds.), Insect pheromones. IOBC Bull. 16, IOBC, Montfavet, France.

Van der Kraan, C., P. Van Deventer et A.K. Minks. 1980. Use of sex pheromones for the control of leafrollers in orchards. Pages 211-214 in : A.K. Minks et P. Gruys (éds.), Integrated control of insect pests in the Netherlands. Pudoc, Wageningen.
Vincent, C. et N.J. Bostanian. 1988. La protection des vergers de pommiers au Québec : état de la question. Nat. Can. (Rev. Écol. Syst.) $115: 261-276$.

Vincent, C., M. Mailloux, E.A.C. Hagley, W.H. Reissig, W.M. Coli, et T.H. Hosmer. 1990. Monitoring the codling moth (Lepidopte$\mathrm{ra}$ : Olethreutidae) and the oblique-banded leafroller (Lepidoptera: Tortricidae) with sticky and non-sticky traps. J. Econ. Entomol. 83 : 434-440.

Welter, S., L. Varela et R. Freeman. 1991. Codling moth resistance in California. Bull. Assoc. Appl. Insect Ecol. 6 : 1-5.

Zlatanova, A.A. 1990. Recovery of populations of codling moth Laspeyresia pomonella L. (Lepidoptera: Tortricidae) after severe reduction due to extreme conditions. Entomol. Rev. 69 : 47-49. 